\title{
Access to Allied Health Care Services in Canadian Interdisciplinary Complex Nerve Injury Programs
}

\author{
Kristine M. Chapman, Chris Doherty, Sean G. Bristol, Russell O’Connor, \\ Michael J. Berger (D)
}

\begin{abstract}
Nerve transfer surgery for patients with nerve and spinal cord injuries can result in dramatic functional improvements. As a result, interdisciplinary complex nerve injury programs (CNIPs) have been established in many Canadian centers, providing electrodiagnostic and surgical consultations in a single encounter. We sought to determine which allied health care services are included in Canadian CNIPs, at the 3rd Annual Canadian Peripheral Nerve Symposium. Twenty CNIPs responded to a brief survey and reported access as follows: occupational therapy $=60 \%$, physiotherapy $=40 \%$, social work $=20 \%$, and mental health $=10 \%$. Access to allied health services is variable in CNIPs across Canada, possibly resulting in heterogeneity in patient care.

RÉSUMÉ : Accéder au Canada à des services de soins de santé connexes dans le cadre de programmes complexes de prise en charge de lésions aux nerfs. Les interventions chirurgicales de transfert des nerfs peuvent, dans le cas de patients victimes de lésions aux nerfs et à la moelle épinière, entraîner des améliorations spectaculaires sur le plan fonctionnel. En conséquence, des programmes interdisciplinaires ciblant ce type de lésions ont été mis sur pied dans de nombreux établissements de santé du Canada. Ces derniers offrent alors des tests électro-diagnostiques et des consultations chirurgicales lors d'une seule rencontre. Lors du troisième Symposium annuel canadien portant sur les nerfs périphériques, nous avons donc cherché à identifier les services de soins de santé connexes qui sont inclus dans ces programmes canadiens. Au total, les représentants de 20 programmes de ce type ont répondu à un bref sondage et ont signalé l'accès aux services suivants : ergothérapie (60\%), physiothérapie (40\%), travail social (20\%) et santé mentale (10\%). À l'échelle canadienne, l'accès à de tels services est en somme variable selon ces programmes, ce qui sous-tend vraisemblablement une hétérogénéité des soins offerts aux patients.
\end{abstract}

Keywords: Peripheral nerve injury, Nerve transfer surgery, Interdisciplinary, Allied health care

doi:10.1017/cjn.2020.98

Can J Neurol Sci. 2020; 47: 830-833

Nerve transfer surgery (NT), involving coaptation of a donor nerve from a functionally redundant muscle group to a paralyzed recipient muscle group, can result in dramatic improvements in muscle strength in patients with upper limb paralysis, secondary to peripheral nerve injuries, spinal cord injuries, and immune-mediated plexopathies. ${ }^{1}$ Interdisciplinary complex nerve injury programs (CNIPs) have recently established at many Canadian centers in response to this paradigm shift in treatment, allowing for electrodiagnostic studies, surgical and rehabilitation planning during a single patient encounter. ${ }^{2}$ The significant care needs of patients presenting to CNIPs also warrant the inclusion of nonphysician professionals into the interdisciplinary team, including occupational therapists (OT), physiotherapists (PT), mental health clinicians (e.g., clinical psychologist; mental health clinician (MHC)), and social workers (SW). Interdisciplinary care has been shown to improve mortality and morbidity in other neurological conditions, ${ }^{3,4}$ so it is important to identify the current breadth of allied health services offered through CNIPs. The purpose of this study was to survey Canadian CNIPs to establish access to allied health services within individual centers, in order to identify gaps in access and regional disparities.

Our survey study was undertaken during the 3rd Annual Canadian Peripheral Nerve Symposium (November 15, 2019, London,
ON). Meeting delegates were invited to complete a brief survey regarding access to allied health services at their respective CNIPs. CNIPs were not identified a priori; therefore, participants were asked to include the name/location of their clinic. Participation was voluntary. Participants were provided with a list of health professions as follows: OT, PT, MHC, and SW. Participants were asked to state whether these services are represented in their clinic, the total number of professionals within each discipline, and whether access is full time or part time. Clinics from large academic centers that were not represented at the meeting were separately contacted by one of the study authors to determine access to allied health.

From the British Columbia Centre for Complex Nerve Injury, Vancouver, British Columbia, Canada (KMC, CD, SGB, RO, MJB); Division of Neurology, Department of Medicine, University of British Columbia, Vancouver, British Columbia, Canada (KMC); Division of Plastic Surgery, Department of Surgery, University of British Columbia, Vancouver, British Columbia, Canada (CD); Division of Surgery, Department of Medicine, University of British Columbia, Vancouver, British Columbia, Canada (SGB); Division of Physical Medicine \& Rehabilitation, Department of Medicine, University of British Columbia, Vancouver, British Columbia, Canada (RO, MJB); and ICORD-Blusson Spinal Cord Centre, Vancouver, British Columbia, Canada (MJB)

Received March 31, 2020. Final Revisions Submitted April 29, 2020. Date of AcCeptance May 17, 2020.

Correspondence to: Michael J. Berger, ICORD-Blusson Spinal Cord Centre, 818 West 10th Avenue, Vancouver, British Columbia, Canada, V5Z 1M9. Email: Michael. Berger@vch.ca 


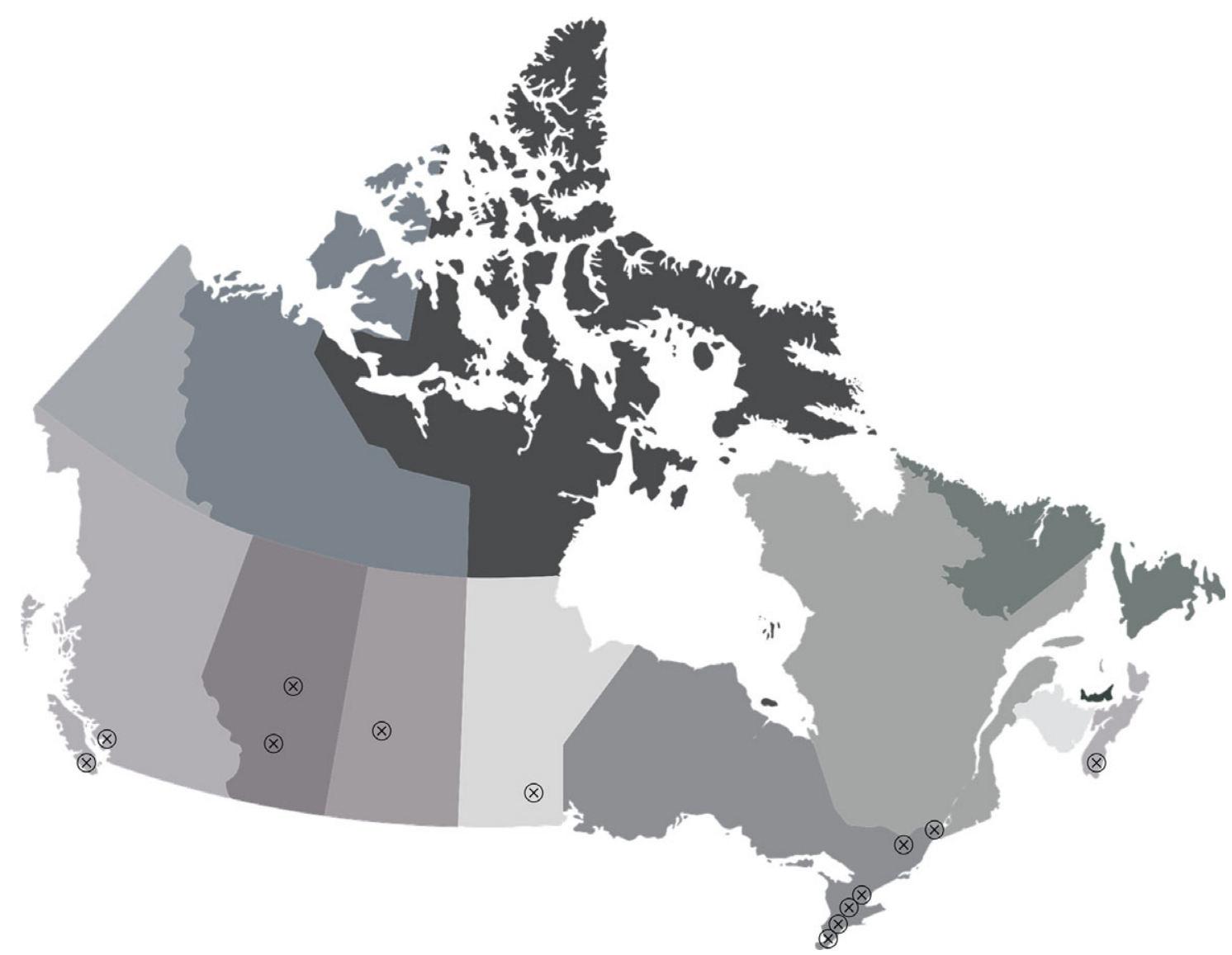

Figure 1: Geographic representation of survey respondents. It should be noted that multiple complex nerve injury programs exist in Toronto and Hamilton, Ontario, Canada.

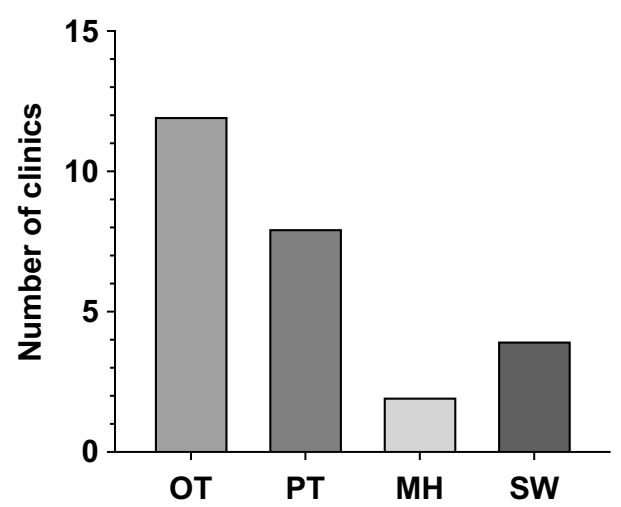

Figure 2: Number of clinics with access to specific allied health care services. A total of 20 clinics responded to the survey.

Twenty programs responded to the survey (13 adult academic centers, 3 pediatric academic centers, 3 regional/community programs, and 1 provincial worker's compensation board). The geographic representation of respondents is presented in Figure 1. Program responses regarding access to each allied health profession are presented in Figure 2. Allotment of access was dichotomous, in the sense that a program was deemed to have access to a service, regardless of whether it is available full or part time. OT was the most frequently reported allied health service available in $60 \%$ of programs; however, respondents noted variable access to
OT, with a range of 0.125 (\% full-time equivalents) - 3 full-time OTs. PT (available at $40 \%$ of the program) was accessible within a range of 0.125 (\% full-time equivalents) -2 full-time PTs. Eight programs $(40 \%)$ described no access to allied health services. One program described access to all health services listed on the survey.

The results of this survey study demonstrate that Canadian CNIPs have variable, and overall, limited access to allied health services. The results highlight the potential for regional disparity in patient access to interdisciplinary care. Interdisciplinary clinics are defined as "a group of health care professionals who have cognitive and procedural expertise in different areas of care delivery and can efficiently manage complex medical conditions." ${ }^{\text {}}$ The benefits of interdisciplinary clinics on mortality and morbidity outcomes in other neurological conditions are well established. ${ }^{3,4}$

Given these known benefits, the results of our study are surprising. Access to allied health services is limited in many CNIPs and not available at all in others. This constitutes a significant gap in care for complex nerve injury patients. Outcomes for other surgical interventions such as tendon transfer, nerve grafting, and nerve repair are also likely dependent on access to allied health services. Therefore, the implications of limited and/or variable access to these services have broader implications beyond NT.

Patients presenting to CNIPs have diverse needs. Among the most important aspects is access to rehabilitation. Even before surgery is offered, it is crucial to maintain range of motion to prevent contractures and manage edema and pain. Outcomes after nerve transfer are dependent on intensive rehabilitation to promote 
"donor activation." "In nerve transfer, a nerve or fascicle from a relatively redundant muscle group is co-apted to a denervated recipient nerve, to restore muscle strength. This requires that the initial function of the donor nerve be "repurposed" for a new action, which in turn requires substantial cortical remodeling. In the early stages of rehabilitation, the recipient muscle may only be activated with concomitant activation of the donor muscle. ${ }^{6}$ Learning how to activate the recipient muscle independent of the donor muscle action may take months to years. The complexity of this movement sequence underscores the importance of intensive supervised rehabilitation, in both the pre- and post-operative period. The results of our study suggest that access to supervised rehabilitation by a qualified OT or PT is limited. Only $60 \%$ and $40 \%$ of the programs reported access to OT and PT, respectively (although it should be noted that $8 / 20$ programs reported access to both $\mathrm{OT}$ and $\mathrm{PT}$ and that $8 / 20$ programs reported access to neither). This is concerning, considering the importance of supervised rehabilitation to outcomes after surgery. It should be further noted that, of the programs reporting access to OT and PT, there was a range of weekly access from just over $1 / 2$ day per week to multiple OTs/PTs in a single clinic. Therefore, even if supervised rehabilitation is available, it may not be provided at the optimal frequency and intensity.

Along with the loss of sensorimotor function associated with a complex nerve injury, it is becoming increasingly recognized that other secondary complications may influence patient outcomes, including depression and pain. ${ }^{7,8}$ The prevalence of depression and anxiety and in patients with brachial plexus injuries is reported to be as high as $38 \%$ and $42 \%$, respectively. ${ }^{7}$ Pain is also highly prevalent in patients with brachial plexus injuries, reportedly as high as $69 \% .^{8}$ MHCs (e.g., psychiatrist or clinical psychologist) would be well suited to mitigate these secondary complications. However, only a single CNIP reported access to MHC, suggesting that these secondary complications could be undertreated.

Finally, loss of pre-morbid vocation following severe nerve injury may be as high as $50 \% .^{9}$ In this regard, it would be helpful to have access to SW or other disability advocate to optimize disability benefits and resources. Only $12 \%$ of programs reported access to SW in their clinics.

Nerve injuries disproportionately affect younger, workingaged individuals. ${ }^{10}$ The economic burden of complex nerve injuries has not been examined in Canada, but US data indicate that the indirect lifetime economic impact of brachial plexus injuries is $\sim \$ 800 \mathrm{~K}$ per patient. ${ }^{10}$ Suboptimal rehabilitation could influence health economic indices such as return to employment and cost of future care. We speculate that systematic inclusion of allied health services in Canadian CNIPs would positively influence economic indices, but this would require a Canadian-based health economic analysis of complex nerve injuries.

This study constitutes a preliminary investigation regarding access to allied health services for complex nerve injury patients and therefore has several limitations. First, although many programs were represented in the survey, our results may not encompass the experience of all programs that exist in Canada (i.e., it includes only those who were represented at the symposium and large regional academic colleagues who are widely known, but were not represented at the meeting). Second, while our results highlight limited resources for allied health services at many CNIPs, they do not address the reasons underlying limited resource allocation, which could potentially include size of clinic catchment area, duration of clinic existence, availability of expertise, and variability in health authority funding. A separate in-depth analysis is required to further understand the reasons for the observed variability. Third, patients experiencing limb paralysis in Canada may have access to allied health services through other means (e.g., third-party insurance and worker's compensation), and this was not reflected in the survey. A future study would examine the experiences of individual patients enrolled in CNIPs to obtain a more comprehensive illustration of access to allied health. Last, out study did not capture the intensity and duration of therapy follow-up in individual patients, within each center. As the post-operative rehabilitation needs of each patient are significant and lengthy (e.g., some patients require 1-2 years of follow-up with therapy), it is possible that despite access to therapy, individual patients may be receiving inadequate intensity and length of follow-up. It is probable that building expertise among general therapists in the community setting would address this potential gap, but this requires a separate and more detailed assessment of therapy intensity within individual centers.

Interdisciplinary clinics are known to improve outcomes. For patients presenting to Canadian CNIPs, care may be enhanced by access to allied health care services to optimize rehabilitation and mitigate secondary complications. Our results demonstrate regional disparity in access to these services and limited access overall. These results will be valuable in advocating for further resources to provide comprehensive clinical services for patients with complex nerve injuries. Questions about rehabilitation intensity and length of follow-up still remain and require future study to further address optimal resource availability within individual centers.

\section{Disclosures}

None.

\section{Statement of Authorship}

KMC was responsible for study design, data collection and analysis, and drafting of the manuscript. CD was responsible for study design, data collection and analysis, and drafting of the manuscript. SGB was responsible for study design. RO was responsible for study design and data collection. MJB was responsible for data analysis and drafting of the manuscript. All study authors were responsible for final approval of the manuscript.

\section{REFERENCES}

1. Moore AM, Novak CB. Advances in nerve transfer surgery. J Hand Ther. 2014;27:96-104; quiz 5.

2. Korus L, Ross DC, Doherty CD, Miller TA. Nerve transfers and neurotization in peripheral nerve injury, from surgery to rehabilitation. J Neurol Neurosurg Psychiatry 2016;87:188-97.

3. Qamar MA, Harington G, Trump S, Johnson J, Roberts F, Frost E. Multidisciplinary care in Parkinson's disease. Int Rev Neurobiol. 2017;132:511-23.

4. Van den Berg JP, Kalmijn S, Lindeman E, et al. Multidisciplinary ALS care improves quality of life in patients with ALS. Neurology $2005 ; 65: 1264-7$. 
5. Tyler KH, Haverkos BM, Hastings J, et al. The role of an integrated multidisciplinary clinic in the management of patients with cutaneous lymphoma. Front Oncol 2015;5:136.

6. Novak CB, von der Heyde RL. Rehabilitation of the upper extremity following nerve and tendon reconstruction: when and how. Semin Plast Surg 2015;29:73-80.

7. Yannascoli SM, Stwalley D, Saeed MJ, Olsen MA, Dy CJ. A population-based assessment of depression and anxiety in patients with brachial plexus injuries. J Hand Surg Am. 2018;43:1136 e1-e9.
8. Ciaramitaro P, Padua L, Devigili G, et al. Prevalence of neuropathic pain in patients with traumatic brachial plexus injury: a multicenter prospective hospital-based study. Pain Med 2017;18:2428-32.

9. Kretschmer T, Ihle S, Antoniadis G, et al. Patient satisfaction and disability after brachial plexus surgery. Neurosurgery 2009;65: A189-96.

10. Hong TS, Tian A, Sachar R, Ray WZ, Brogan DM, Dy CJ. Indirect cost of traumatic brachial plexus injuries in the United States. J Bone Joint Surg Am. 2019;101:e80. 